\title{
Analyse de la situation météorologique à l'origine des crues des 3 et 4 octobre 1960 dans le nord-ouest du Massif Central
}

Définition des types de situations météorologiques provoquant des crues sur les rivières issues du plateau de Millevaches

\section{Analysis of the meteorological conditions responsible for the 3rd and 4th October floods in the north-western Central Massif}

Definition of the meteorological conditions causing spate conditions in the rivers issuing from the Millevaches plateau.

PAR P. FON'TATNE

INGÉNIEUR EN CHEF DE LA MÉTÉOROLOGIE

\begin{abstract}
Les pluies intenses qui ont conduit aux crues exceptionnelles des 3 et 4 octobre 1960 dans le nord-ouest du Massif Central ont été provoquées par le conflit d'un courant chaud, d'abord tropical marifime puis méditerranéen, avec des masses d'air froid stagnant sur le centre de la France, notamment sur le plateau de Millevaches dont l'effet orographique a été ainsi accentué.

Cette situation météorologique a présenté initialement beaucoup d'analogie avec celle qui est habituellement observée lors d'importantes crues cévenoles. Toutefois elle en a différé par une position notablement plus occidentale du courant humide et chaud.

Il s'agit d'un cas très rare, ce que confirme sa comparaison avec les types de situations météorologiques qui ont engendré au cours des quarante dernières années des crues sur des rivières telles que la Corrèze et la Creuse.
\end{abstract}

\begin{abstract}
The intense rainfall that caused the exceptional floods experienced in the north-western Central Massif on the 3rd and 4th October 1960 was due to the meeting of an (originally tropical maritime, then Mediterranean) warm, air stream with stationary cold air masses over central France or, more precisely, over the Millevaches plateau, the orographic effect of which was thus greatly enhanced.

At first, these meteorological conditions were very similar to those normally associated with major floods in the Cévennes hills, except that, this time, the warm air stream lay much further to the west.

That such meteorological conditions were quite exceptional was confirmed when they were compared with those responsible for floods in such rivers as the Correze and Crense during the past 40 years.
\end{abstract}

\section{I. - EVOLUTION DE LA SITUATION METEOROLOGIQUE ENTRE LE 30 SEPTEMBRE ET LE 4 OCTOBRE 1960}

\section{I.1. Phases préliminaires et principales.}

Dans une phase préliminaire, relative aux journées du 30 septembre et du $1^{\text {er }}$ octobre, la constitution d'un dôme d'air froid d'une épaisseur d'environ 5000 mètres, du nord de l'Italie à la Suisse, revêt une grande importance.
En effet, une nouvelle perturbation pluvieuse, qui aborde la France et la Péninsule Ibérique au début de la journée du 2 octobre (fig. 1) est fortement ralentie par suite du «blocage» dû à cette masse d'air froid, gui tend d'ailleurs à s'écouler en partie vers la Provence et la vallée du Rhône. De ce fait, à la fin de la journée du 


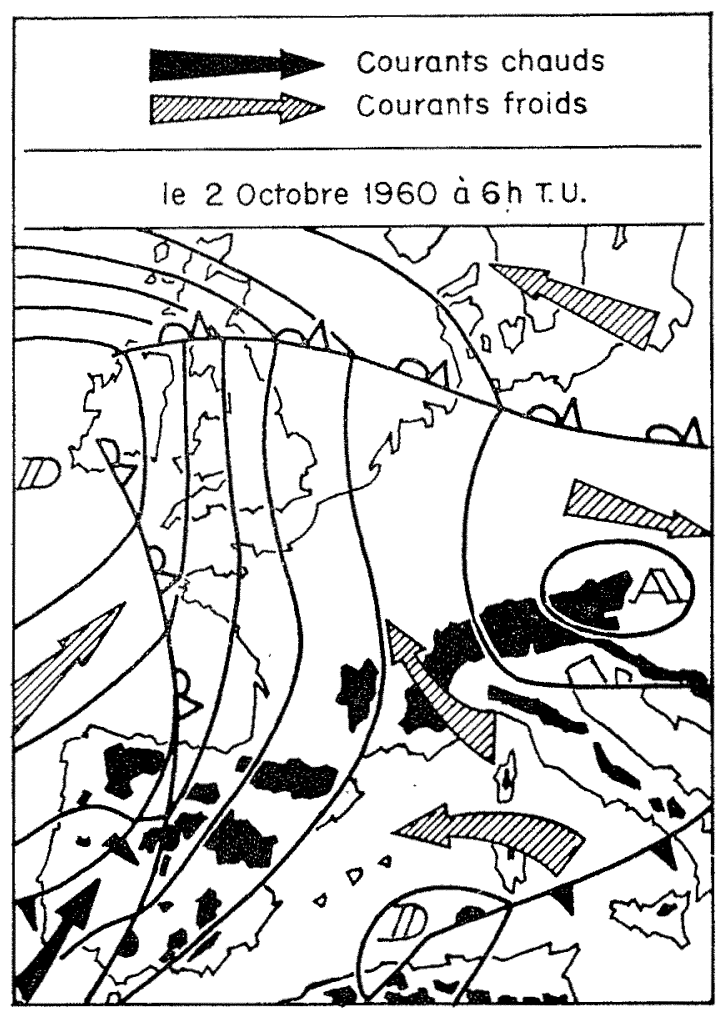

FIG. 1

2 octobre, les pluies n'ont guère dépassė les régions voisines de l'Atlantique.

Mais un autre facteur de ralentissement apparaît au début de la journée du 3 octobre avec l'affaiblissement soudain $d u$ courant polaire maritime, à composante ouest, régnant sur le

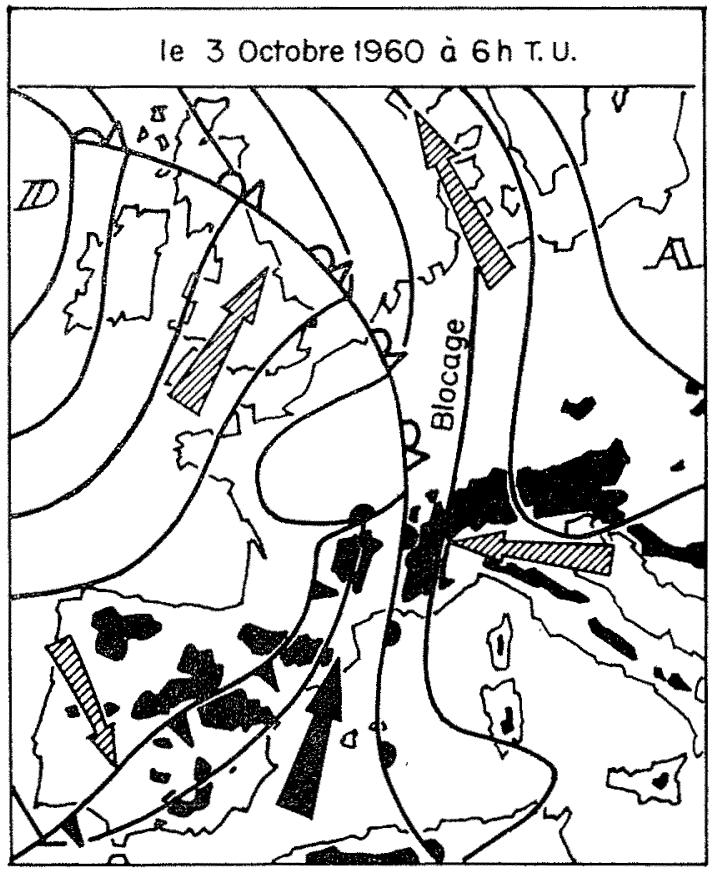

Frg. 2 proche Océan (fig. 2), Cet affaiblissement, dont les causes sont complexes, a pour effets de permettre un regain d'activité du courant tropical océanique constituant le secteur chaud de la perturbation, et d'enrayer le phénomène habituel d'occlusion.

Dans ces conditions, le courant tropical océanique, après avoir franchi les Pyrénées, commence à intéresser le sud du Bassin Aquitain au début de l'après-midi du 3 octobre. Ainsi débute la phase terminale de l'évolution qui conduira dans les douze heures suivantes à des précipitations exceptionnelles sur le nord-ouest du Massif Central. Mais, avant d'examiner plus particulièrement cette courte période, on doit signaler l'analogie que présentait initialement la situation météorologique du 3 octobre 1960 avec les situations des 30 septembre et 4 octobre 1958, qui furent, on s'en souvient à l'origine de grandes crues cévenoles.

1.2. Analogie entre la situation météorologique du 3 octobre 1960 et celle du 4 octobre 1958. Critères à retenir pour différencier les situations, apparem. ment analogues, responsables de fortes précipitations soit sur les Cévennes, soit sur le nord-ouest du Massif Central.

L'analogie, déjà bonne avec la situation du 30 septembre 1958, fut surtout remarquable avec celle du 4 octobre 1958 (fig. 3). En effet,

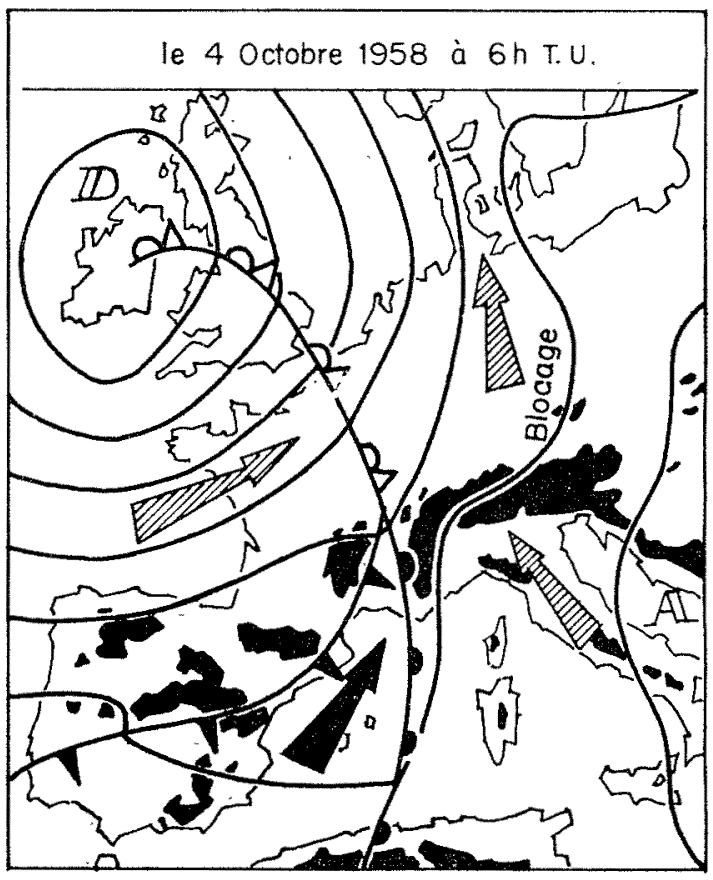

Fig. 3 
hormis une similitude de dates presque parfaite, on décelait dans les deux cas: un centre d'action dépressionnaire voisin de l'Irlande prolongé par un thalweg en direction des côtes du Portugal, une perturbation d'origine atlantique alimentée en air tropical maritime puis méditerranéen rejetée vers l'Est par le courant polaire maritime froid circulant autour de la dépression d'Irlande, enfin, relié à un anticyclone continental, un «coin» d'air froid suffisamment avancé vers l'ouest pour constituer une zone de «blocage $»$ pour la perturbation venue de l'Atlantique. Celte énumération des caractéristiques communes aux situations météorologiques des 3 octobre 1960 et 4 octobre 1958 concorde parfaitement avec la description du type de temps à fortes crues cévenoles faite dans des études antérieures relatives à ces phénomènes $\left(^{*}\right)$.

Quels sont alors les facteurs moins directement apparents qui ont conduit dans le cas particulier du 3 octobre 1960, à une position notablement plus occidentale du maximum pluviométrique et, par voie de conséquence, à des crues spectaculaires à une époque assez inhabituelle de l'année dans le nord-ouest du Massif Central, au lieu des Cévennes comme on pouvait logiquement le craindre. Ces facteurs, qui en général ne peuvent être décelés qu'assez tardivement, se rapportaient surtout à l'importance de la masse d'air continental froid (5000 m d'épaisseur le 3 octobre 1960 au lieu de $3500 \mathrm{~m}$ le 4 octobre 1958) et à son extension vers l'ouest qui fut maximale le 3 octobre 1960. L'atténuation subite du courant atlantique froid, qui ne s'était pratiquement pas manifestée le 4 octobre 1958 , est également un facteur à retenir pour que d'importantes précipitations puissent se produire sur le nord-ouest du Massif Central.

\section{I.3. Phase terminale, relative à l'évolution de la situation météorologique entre le 30 septembre et le 4 octobre 1960.}

Comme il a été dit, au début de cet exposé, le courant très humide, d'origine tropicale océanique, constituant le secteur chaud de la perturbation responsable des crues en cause, aborde le sud du Bassin Aquitain au début de l'aprèsmidi du 3 octobre 1960. En effet, ce courant n'a pu se décaler plus vers l'est à cause du «blocage » d̂̀ à l'air continental relativement froid qui s'est avancé au moins jusqu'à la vallée du Rhône, et à cause aussi du manque de vigueur du courant atlantique postérieur à la perturba-

(*) Climat pluviométrique des Basses-Cévennes, par R. Rebotier. Monographie $n^{0} 7$ de la Météorologie nationale. - Causes météorologiques des grandes crues cévenoles du début de l'automne 1958, par P. Fontaine et G. Portela, la Météorologie, janvier-mars 1959. tion. Maintenant, par vagues successives, et à l'ouest de la ligne de partage des eaux, le courant chaud progresse vers le nord à la rencontre de masses d'air plus frais recouvrant le nord, l'ouest et le centre de la France (fig. 4). Une zone de conflit entre l'air chaud et l'air froid apparaît dès la soirée du 3 octobre sur les régions s'étendant du Bordelais aux Monts Dômes avec une intensité maximale sur le cours supérieur de l'Isle, de la Vézère et de la Corrèze. Plus tard, après une accalmie toute relative, le courant chaud et humide, qui a évolué vers le type méditerranéen et qui s'est légèrement décalé vers l'est sans toutefois pouvoir trouver d'autre issue vers le nord que le Seuil de Naurouze, marque un regain.d'activité dans la nuit du 3 au 4 octobre, conduisant ainsi à une recrudescence du conflit avec l'air froid, particulièrement résistant dans la zone du plateau de Millevaches.

Les maximums d'intensité des précipitations, enregistrées par les stations pluviométriques d'E.D.F., d'une part entre 16 heures et 19 heures le 3 octobre, d'autre part entre 21 heures le 3 et 4 heures le 4 octobre, confirment cette évoluIution.

En conclusion de ce chapitre, on remarquera que seule l'analyse tridimensionnelle des masses d'air peut fournir l'explication de l'importance

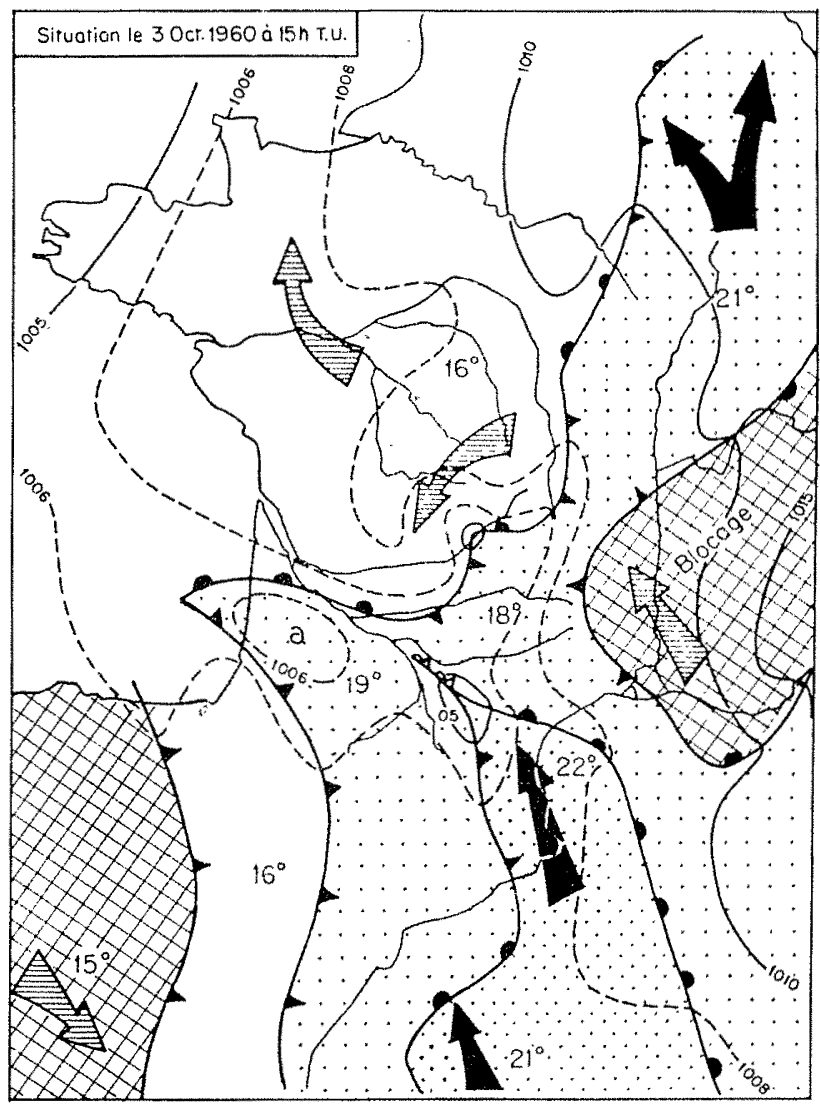

FIG. 4 
des précipitations dans la zone où se sont produites les crues. Car le relief, par trop médiocre, ne peut être mis seul en cause. Par contre, recouvert, comme nous l'avons montré, d'un dôme d'air froid peu mobile, il a constitué un obstacle très important pour le courant chaud et humide et à partir de l'ascendance créée par ce relief, artificiellement et passagèrement «renforcé», les importantes précipitations observées s'expli- quent facilement. En particulier, un calcul simple basé sur les équations appropriées de thermodynamique permet une estimation de l'intensité moyenne horaire des précipitations. La valeur ainsi trouvée $(8,4 \mathrm{~mm} / \mathrm{h})$ se révèle très voisine de celle déduite des pluviogrammes $(9,6 \mathrm{~mm} / \mathrm{h})$, ce qui apporte un argument supplémentaire en faveur de l'évolution des masses d'air telle qu'elle a été décrite ci-dessus.

\section{II. - DÉFINITION DES TYPES DE SITUATIONS MÉTÉOROLOGIQUES PROVOQUANT DES CRUES SUR LES RIVIËRES ISSUES DU PLATEAU DE MILLEVACHES}

L'intérêt résultant de la définition des types de situations météorologiques responsables de crues dans l'ouest et le nord-ouest du Massif Central est évident. En effet, hormis l'aide qu'en peuvent attendre les météorologistes chargés de prévoir ces situations et d'en informer les services intéressés, cette classification permet, comme on le verra ci-après, de se faire une opinion sur la fréquence probable de retour d'une situation analogue à celle du 3 octobre 1960 .

Donc, sur la base de données hydrométriques, relatives aux crues de la Corrèze à Brive et de la Creuse à Argenton et au Blanc, et se rapportant à la période 1919-1960, 24 situations météorologiques, correspondant à ces crues, ont été

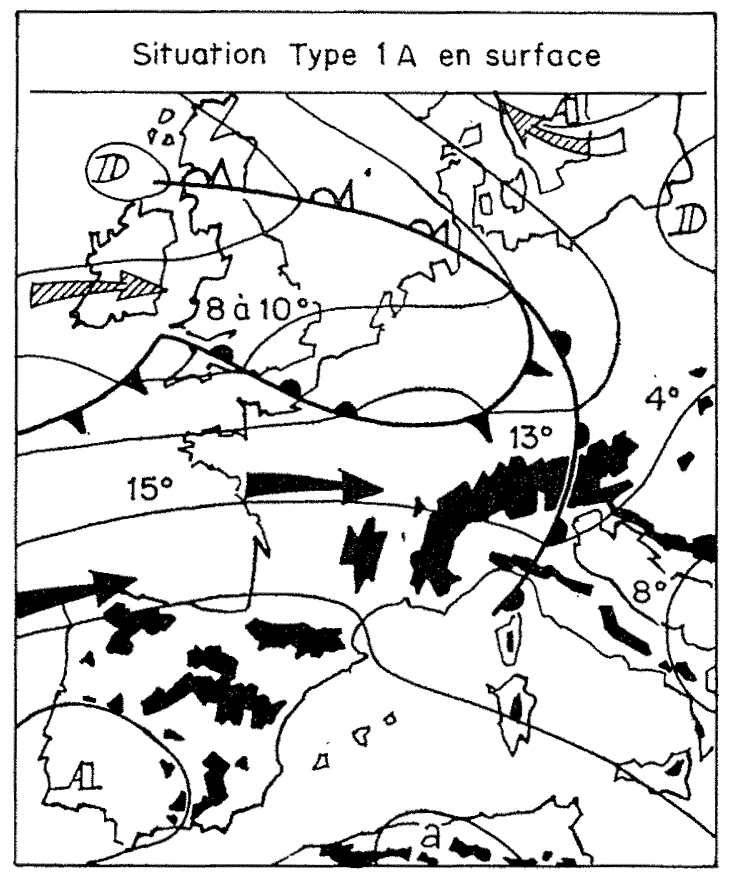

FIG. 5 examinées et classées. On a pu en extraire six situations types se définissant ainsi :

1. Les circulations zonales à composante ouest comprenant :

1.A les advections d'air chaud d'origine tropicale océanique;

1.B les familles de perturbations d'ouest;

1.C les advections d'air froid d'origine polaire ou arctique maritime.

2. Les circulations méridiennes à composante sud :

2.A avec prépondérance de l'influence méditerranéenne;

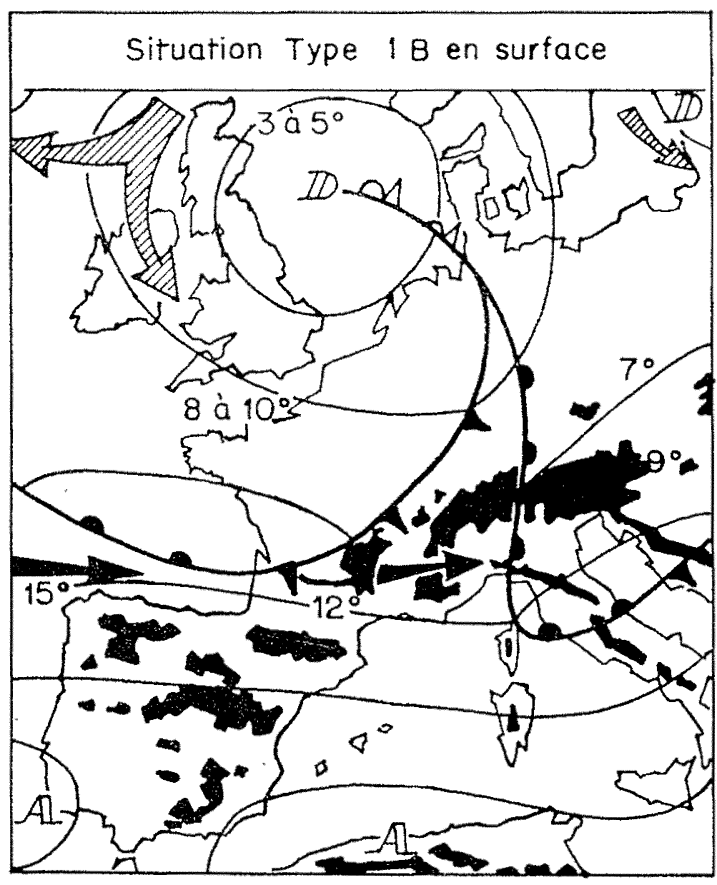

FIG. 6 


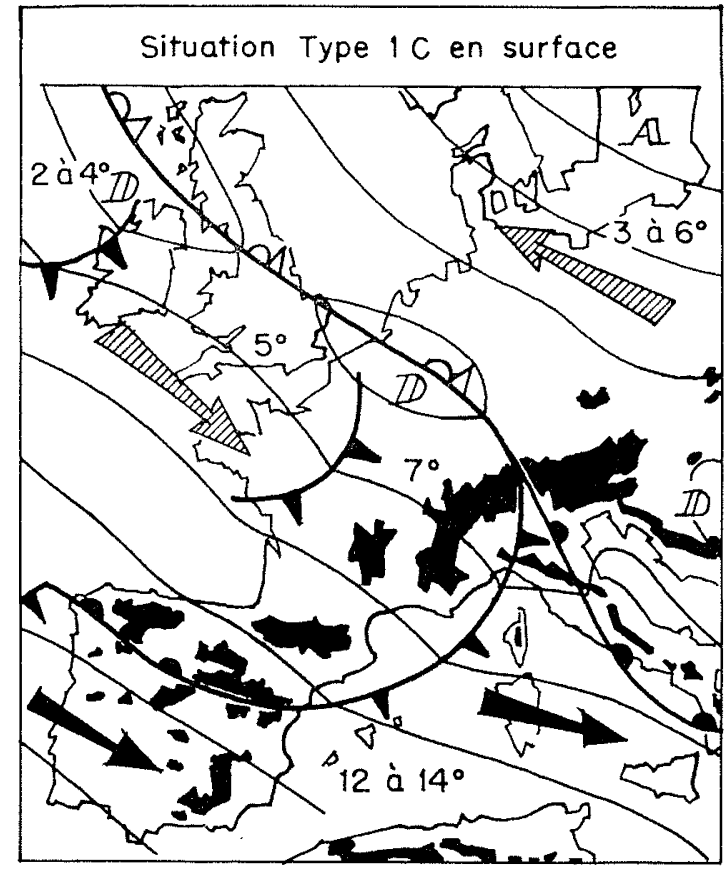

FIG. 7

2.B avec partage des influences méditerranéennes et océaniques.

3. Les circulations mal définies ou marais barométriques :

Pour chacune de ces six situations types, il a été établi des schémas relatifs au champ de pression en surface (fig. 5 à 10) et en altitude, ainsi qu'une fiche «signalétique» comportant de

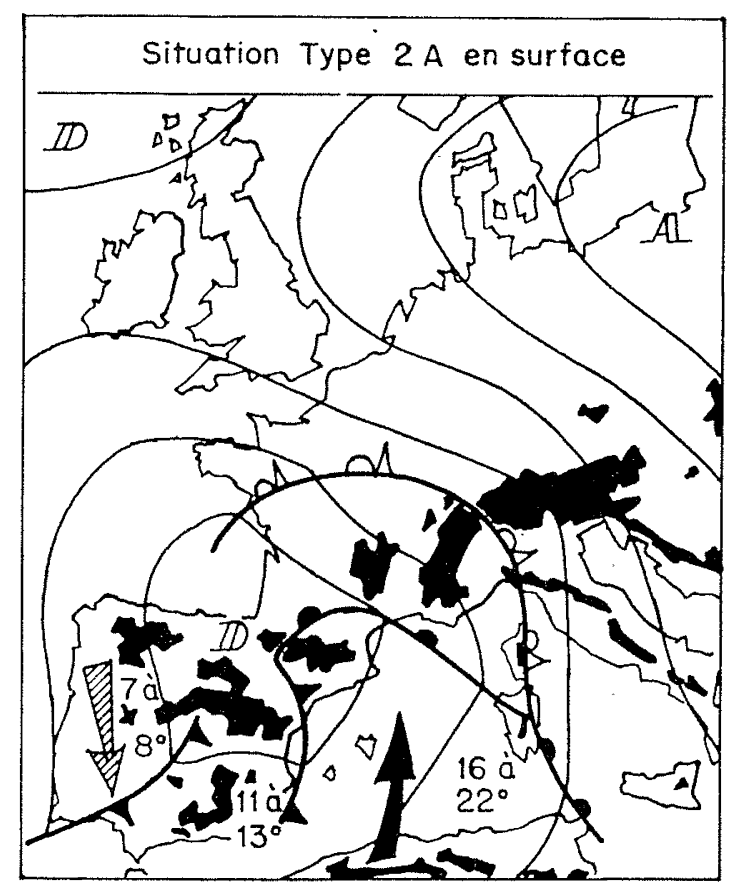

FIG. 8

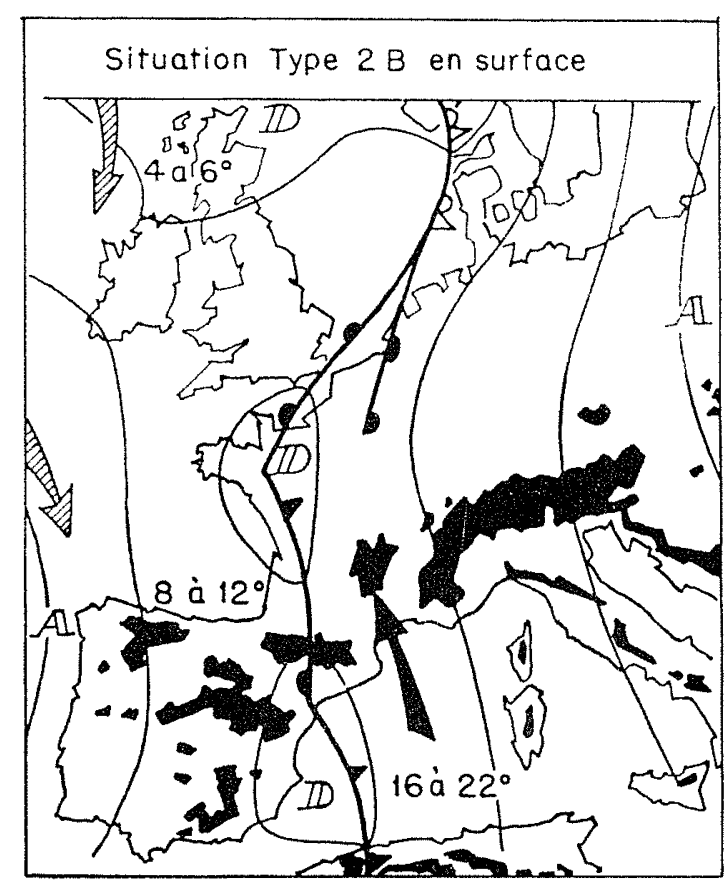

Fig. 9

nombreuses indications supplémentaires se rapportant, notamment, aux plus fortes précipitations, à l'influence nivale et aux caractères généraux des crues. Mais la place disponible pour cette étude n'a pas permis la publication de ces fiches ni des situations en altitude. Ces renseignements complémentaires pourront toutefois être communiquès, sur leur demande, aux personnes intéressées par ces questions.

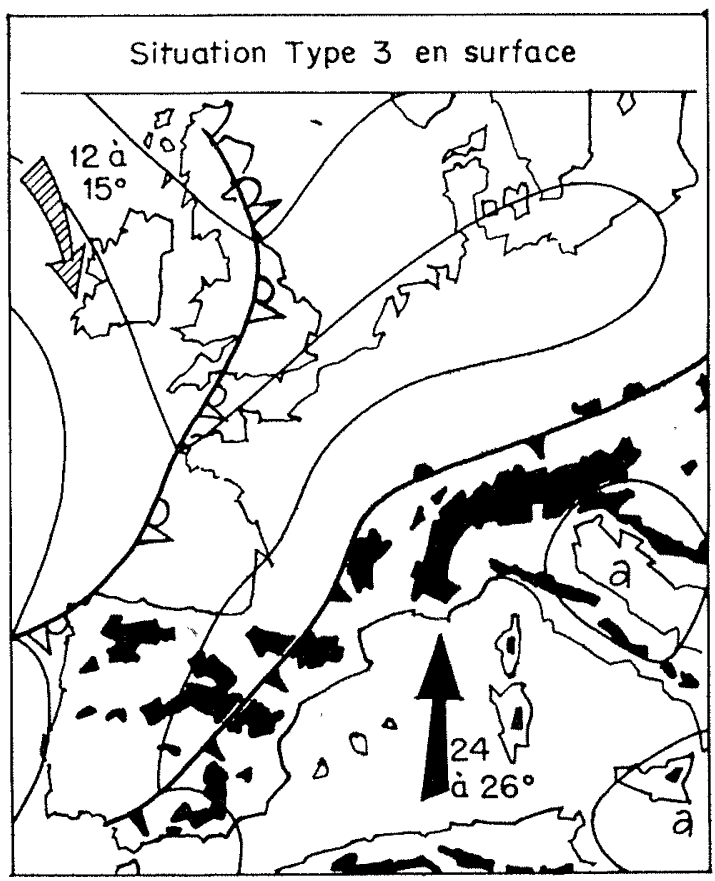

Fig. 10 


\section{III. - POSITION DE LA SITUATION MÉTÉOROLOGIQUE A L'ORIGINE DES CRUES DES 3 ET 4 OCTOBRE 1960 PAR RAPPORT AUX SITUATIONS TYPES}

Telle qu'elle a été définie et schématisée dans les précédents chapitres, on voit aisément que cette situation ne présente que peu d'analogie avec l'une quelconque des situations types, bien que se rapportant indiscutablement à la catégorie générale des situations caractérisées par une circulation méridienne à composante sud.

Il s'agit donc d'un cas hors série, constituant une variante extrêmement rare d'un des types de situation engendrant habituellement des crues cévenoles.

L'intérêt de cette constatation est qu'elle confirme le caractère exceptionnel des crues observées les 3 et 4 octobre 1960 , ce qui ressortait déjà d'analyses statistiques portant sur les précipitations maximales et sur les débits maximaux.

\section{IV. - CONCLUSIONS}

La prévision des débits et l'annonce des crues deviendraient considérablement plus efficaces si elles pouvaient être réalisées à partir de prévisions quantitatives de précipitations dont l'exactitude s'inscrirait dans des limites acceptables. Mais la réalisation de telles prévisions, à partir de la thermodynamique, est rendue fort difficile par le fait que les éléments à prendre en considération pour déterminer la répartition des masses d'air et leurs actions réciproques sur la France, à 12 ou 24 heures d'échéance, sont alors situés à plus de 1000 ou $1500 \mathrm{~km}$, c'est-à-dire sur l'Atlantique ou sur la Méditerranée qui sont des régions de moindre densité pour les renseignements météorologiques.

Pour autant, la Météorologie Nationale ne se désintéresse pas de ces problèmes. Au contraire, ils figurent parmi les thèmes d'étude auxquels seront appliquées les possibilités offertes par le calculateur électronique dont ce service dispose.

Mais, dès à présent, d'assez bonnes prévisions de précipitations, portant sur les quantités et les localisations, peuvent être élaborées sur la base d'analogies avec des situations types, définies comme il a été fait dans le cas particulier relatif à la présentc étude.

Des contacts fréquents entre les services intéressés (Météorologie, Annonce des Crues) seront cependant nécessaires à partir du moment où le risque d'établissement d'une situation dangereuse aura été décelée par les météorologistes, car l'annonce en temps opportun de la crue correspondante dépendra pour une part importante de ces contacts.

Une telle collaboration existe déjà pour d'assez nombreux bassins, mais elle demande certainement à être développée et généralisée. La possibilité offerte par la Société Hydrotechnique de France à des ingénieurs des Ponts et Chaussées, du Génie Rural, d'E.D.F. et de la Mínóologie, de réaliser en commun l'étude des récentes crues observées dans le Massif Central préfigure déjà cette collaboration accrue. 


\title{
DISCUSSION
}

\author{
Président: M. DE Rouville
}

M. le Président dégage de l'étude de M. Fontaine qu'il y a une analogie entre les phénomènes qui ont provoqué les crues de 1958 et ceux qui ont provoqué les crues de 1960 : il $y$ a toujours une haute pression de blocage dans l'est qui s'étend plus ou moins au sud, et un centre dépressionnaire depuis l'Irlande.

Subsidiairement, M. le Président, ainsi que M. FonTAINE, préfèrent l'expression de «centre dépressionnaire» à celle de «dépression» parce que la deuxiẻme indique une mobilité plus grande que la première.

Les deux canses sent : des vents chauds venant d'Afrique ou de la Méditerranée, puis des vents de nord-ouest ou du nord qui refroidissent les couches supérieures et enferment parfois la zone de vent chaud entre deux couches froides. C'est ce qui a été dit pour les crues de 1958.

M. Ie Président demande ensuite l'influence, sur le phénomène, du thalweg rattaché à la dépression du nord-ouest de l'Europe et qui s'est allongé vers le sud.

M. Fontarne pense que le thalweg dont parle M. le Président etait à l'origine la trace, sur le champ de pressions, de la perturbation; il y avait de l'aỉr froid à l'ouest et de l'air: froid à l'est. Dans le thalweg, il y a advection d'air chaud. En général, ceci n'apporte pas de grandes précipitations car le thalweg est moins marqué que dans le cas particulier d'octobre 1960 et il se déplace plus rapidement.

M. le Président demande si le courant polaire mentionué dans le rapport de $M$. Fontalne est dû à la dépression ou au courant froid qui peut venir de l'anticyclone de l'est.

M. Fontanse répond que c'est du courant polaire océanique qu'il a parlé.

M. le Président dit que l'on a constaté une analogie entre des crues des Cévennes et celles du plateau de Millevaches; peut-on déceler, à $50 \mathrm{~km}$ près, le siège de la pluie?

M. Fontande croit que cela est possible: on peut prévoir de fortes pluies 24 heures à l'avance et les localiser sur les Cêvennes, comme cela a été fait en octobre 1960 , car le cas du nord-ouest du Massif Central est extrêmement rare; ensuite, par approximations successives, on peut rectifier la prévision à très courtes échéances 12 heures à 6 heures).

D'après une remarque de $M$. le Président disant qu'il y a des inconvénients à alerter des populations émotives qui finiraient ensuite par ne plus s'émouvoir, M. Fonralne croit qu'il est indispensable que le Service Météorologique collabore avee le Service d'Annonce des Crues pour jauger, chacun séparément, le risque et l'annoncer au mieux.

M. le Président ajoute que pour les personnes et les animaux, les grosses catastrophes ne se produisant que quelques heures après que les pluies ont commencé, on a, en gẻnéral, le temps de prévenir les personnes. D'autye part, il peut y avoir des familles de dépression et la succession des périodes plavieuses peut avoir un rythme de 24 heures.

M. le Président demande ensuite à M. Fontarne s'il a quelque chose à ajouter au rapport de M. JACQueT.

M. Fontane dit tout d'abord que M. Jacouer a résumé d'une façon très précise et claire son mémoire et com- plète un point sur lequel lui-même n'a pas assez insisté : l'importance de lair froid postérieur à la periubation; il $\mathrm{y}$ avait eu un affaiblissement très net de l'activité du courant froid: une partie s'est dirigee vers l'Irlande, lautre vers l'Espagne et le Maroc. Cette dernière a provoqué un contre-courant chaud qui a apporté un afflux d'aîr tropical océanique et un afflux d'air méditerranéen. Les causes de cette divergence dans l'air froid océanique sont assez complexes.

M. le Président demande si de grands vents ont accompagné les phénomènes du plateau de Millevaches comme ceux des hautes Cévennes.

M. Fontaine ne croit pas qu'ils aíent été aussi violents.

M. le Président demande si les pluies qui ont progressé du nord-ouest au sud-est dans les Cẻvennes ont eu plus d'action que dans les rivieres divergentes du plateau de Millevaches où elles ont remonté les vallées.

M. Fontane répond que c'est exact et que le reliel a joué beaucoup plus dans le cas de crues cévenoles que dans le cas du platean de Millevaches.

M. Guinot désire présenter une remarque que ferait certainement M. Pardé s'il était présent, et réparer une omission qu'il a luiméme commise dans son exposé sur' les précipitations, en ce sens qu'effectivement il y a eu des pluies cévenoles assez importantes dans la nuit du 3 au 4 octobre 1960. Pour reprendre les termes exacts d'une lettre de M. Pardé : «La tromperie a été tout au plus partielle, puisque cette pluie cévenole eut lieu en nême temps que le déluge Millevaches ou presque».

M. Fontane craint qu'il ne s'agisse pas de la même perturbation.

M. Gurluor confirme qu'il s'agit bien de la même perturbation: l'averse a commencé sur les Cévennes vers $0 \mathrm{~h}$ le 4 et $\mathrm{y}$ a donne, jusque vers $12 \mathrm{~h}, 100 \mathrm{~mm}$ dans $\mathrm{la}$ région des maximums; l'Ardèche a narqué une crue non négligeable dont la pointe est passéc à VallonPont-d'Are vers la fin de la matinée du 4 (1). Ainsi, on ne peut pas dire que les prévisions météorologiques aient été si mauvaises : elles ont simplement péché par omission en ne signalant pas qu'il y aurait renforcement par blocage sur le nord-ouest du Massif Central. A l'inverse, il ne faut pas oublier que le 3 oetobre 1958, $j 1$ $\mathrm{y}$ avait eu des pluies non négligeables sur l'ouest du Massif Central ( $50 \mathrm{~mm}$ et plus) comme suite an balayage régulier par le front froid, l'effet de blocage n'ayant eu lieu qu'ensuite sur les Cévennes.

M. le Président pense que le plus intéressant est de déterminer le point le plus atteint.

M. Batrct.s demande si un parallélisme a èté constaté entre les crues de 1958 et de 1960 et celles du Tarn et de la Garonne au début de mars 1930.

M. le Présídent pense que ces crues n'ont pas eu les mêmes phénomènes : il croit se rappeler qu'elles furcnt

(1) Partant de l'etiage dans la matinée du $A$, Ia pointo

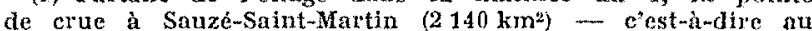
confluent avec le Rhône - a été de $700 \mathrm{~m} / \mathrm{s}$ vers $17 \mathrm{~h}$ le 4 . Aucune confusion possible avee la perturbation suivante, dont les pluies $(160 \mathrm{~mm})$ ont eu lieu $d u 5$ a 4 h au 6 a $6 \mathrm{~h}$; le debit a Sauzé, apres être retombé $2000 \mathrm{~m}^{\mathrm{s}} / \mathrm{s}$ le 5 a $17 \mathrm{~h}$, a marquè une deuxieme pointe de $3300 \mathrm{~m}^{3} / \mathrm{s}$ le 6 iे $6 \mathrm{~h}$. 
dues, au moins partiellement, à une fonte des neiges dans les monts de Lacaune (haute vallée de l'Agout).

M. Fontaine ajoute que ces crues ont dû se faire sentir aussi sur la Corrèze et la Creuse, peut-être avec moins d'importance. Elles se rattachent à des courants méridiens à composantes sud.

M. le Président demande si la répartition des anticyclones était un peu de la même nature.

M. Fontaine indique que, dans cette situation-là, il s'agissait d'une dépression qui se forme sur le bassin méditerranéen et remonte progressivement vers le nordouest de l'Europe. Finalement cela ressemble un peu $\grave{a}$ la situation d'octobre 1960 , mais l'origine des centres d'action n'est absolument pas la même.

A la suite d'une autre question de M. le Président, M. Fontaine explique qu'une dépression qui remonte de la. Méditerranée vers le nord-ouest est plus fréquemment génératrice de crue sur le plateau de Millevaches qu'une situation avec dépression près de l'Irlande.

M. Remenieras demande à M. Fontanne si l'observation par radar de la progression des perturbations pluvieuses ne permettrait pas d'accroître la précision de la localisation des zones menacées par des averses de grande intensité et ne donnerait pas un laps de temps suffisant pour que l'alerte des populations intéressées soit efficace.

M. Fontanne précise qu'il est certain que l'utilisation des radars, qui enregistrent les échos de précipitations de façon très nette, devrait apporter une amélioration très sensible dans la prévision à courte échéance, que la portée des radars dont il dispose est théoriquement de l'ordre de $200 \mathrm{~km}$ et étant donné le déplacement des perturbations, à $50 \mathrm{~km} / \mathrm{h}$ en moyenne, cela fait quatre heures de répit.

M. Remenieras pense que le seul moyen nouveau, proposé depuis trente ans, pour rendre l'alerte efficace dans le cas de petits bassins versants à faible «temps de concentration 》, est le radar météorologique. Il sera particulièrement intéressant de suivre les résultats obtenus par les essais poursuivis dans ce sens, notamment aux Etats-Unis.

M. le Président dit que, lorsque les pluies tombent sur terre, le système du téléphone serait peut-être plus rapide que le radar. Ce qui en ce moment augmente le temps perdu pour la transmission est le fait que les petits pluviomètres ne sont observés que rarement $(6,12$ ou 24 heures). Quand on s'en rend compte, il est déjà tómbé quelques décimètres d'eau.

M. Rementeras pense que l'on pourra résoudre ce problème grâce aux pluviomètres parlants que l'on peut interroger par téléphone ou par radio depuis un poste central.

M. le Président estime que la radio et l'automobile sont des moyens plus" sûrs pour prévenir, car les fils téléphoniques ou télégraphiques sont souvent coupẻs avant que l'on puisse s'en servir pour transmettre.

M. Rementeras observe qu'en périodes très orageuses, les transmissions radio peuvent être très perturbées, en montagne notamment.

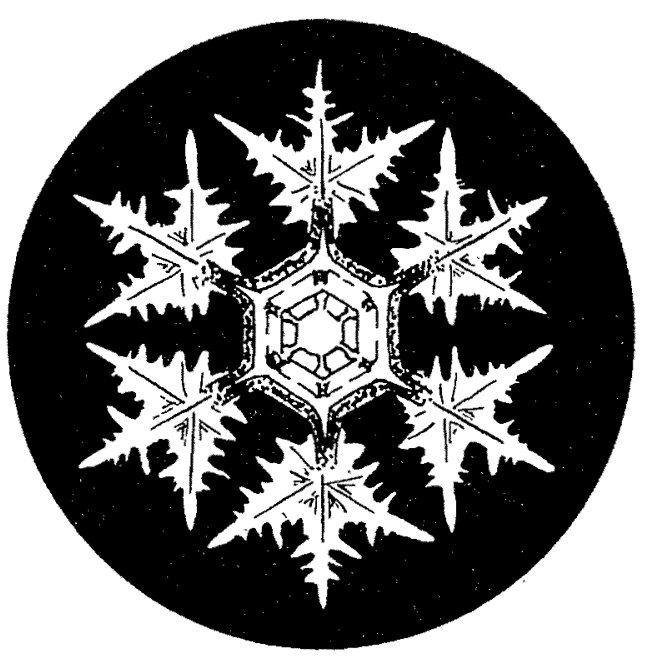

